\title{
Layer-by-Layer Films from Wine: An Investigation of an Exponential Growth Process
}

\author{
Marcio N. Gomes, Jackeline B. Brito, Josmary R. Silva, and Nara C. de Souza \\ Grupo de Materiais Nanoestruturados, Campus Universitário do Araguaia, Universidade Federal de Mato Grosso, \\ Barra do Garças, MT, Brazil \\ Correspondence should be addressed to Nara C. de Souza; ncsouza@ufmt.br
}

Received 17 January 2013; Accepted 5 March 2013

Academic Editor: Shuangxi Xing

Copyright (C) 2013 Marcio N. Gomes et al. This is an open access article distributed under the Creative Commons Attribution License, which permits unrestricted use, distribution, and reproduction in any medium, provided the original work is properly cited.

\begin{abstract}
We report on the preparation and study of layer-by-layer films of wine alternated with bovine serum albumin (BSA). We found that the exponential and/or linear growth of the films is dependent on the deposition time. Atomic force microscopy images were analysed using scale laws and the fractal dimension, and the results suggested that the BSA/wine film growth regime is determined by sub-bilayer or bilayer growth. Exponential growth was associated with a sub-bilayer deposition regime, whereas linear growth was associated with a bilayer deposition in which a constant amount of material is deposited.
\end{abstract}

\section{Introduction}

Grapes and wine have many constituents, such as proteins, sugars, and vitamins, that have nutritional value and thus improve quality of life $[1,2]$. Extensive research has examined the beneficial effects of moderate wine intake, which provides antioxidant effects, decreases levels of low density lipoprotein (LDL), and prevents diabetes, cardiovascular diseases, and neurodegenerative diseases $[3,4]$. Although grapes and wine have similar phytotherapic effects, the literature indicates that wine has enhanced therapeutic properties due to the fermentation process, likely as a result of alcohol production, which improves the vasodilator properties of wine [4].

Among the molecules identified in wine, resveratrol and quercetin (flavonol) are widely investigated because they have anti-inflammatory, antifungal, cardioprotective $[5,6]$, and antitumor properties [7]. Previous research [8-12] has studied the biomedical characteristics of these two molecules in order to correlate their properties with the beneficial effects of moderate wine intake $[3,5]$.

Because of the complexity of the various compounds identified in wine, most studies have investigated isolated molecular groups in an aqueous or alcoholic solution [8, 10]. Most studies investigating wine have characterised the processes of winemaking and its chemical composition [13-17]. More recent work has investigated the interaction of wine with various ligands $[3,18,19]$; however, the mechanisms responsible for these interactions are not further explained.

Films obtained from the layer-by-layer technique (LbL) enable the immobilisation of different molecules on a single substrate and allow the characterisation of a complex multifunctional surface [20]. Two kinds of growth regimes during the build-up of LbL films have been reported: linear growth and exponential growth. For many years, linear growth was the only regime reported $[20,21]$ for the LbL technique. However, in 1999, Elbert et al. reported a ground-breaking discovery in the LbL technique, observing an exponential growth of LbL films from poly(L-lysine) (PLL) alternated with sodium alginate (Alg) [22]. To explain the exponential growth, the authors hypothesised that the increase in the amount of deposited material in each layer was caused by an increase in the film roughness, which was associated with the available deposition area and a fixed number of deposition sites for each area [22]. Since the report of Elbert et al., several systems have been shown to exhibit exponential growth, that is, PLL/hyaluronan [23, 24], PLL/poly(L-glutamic acid) [25], Chitosan/HA [26, 27], and PAH/poly(L-glutamic acid) [28]. 
Interestingly, a regime transition from linear to exponential has been noted to change the ionic strength, polydispersivity, and temperature of the films [29].

Despite the numerous investigations to date, all previous studies on wine have examined fabricated wine (in the liquid phase), and to the best our knowledge, there are no studies on LbL films from wine. The advantage of using the film form of wine components is that several solid state experimental techniques can be used for characterisation. In addition, LbL films based on wine and BSA protein emerge as an experimental model for studies on the interaction between wine components and protein. Polyphenols in wine are capable of forming stable combinations with proteins. Although various types of interactions have been observed, their mechanisms have not been completely explained [30]. The two main types are hydrophobic interactions [31] and hydrogen bonds [32], while ionic or covalent bonds are probably present but less important. In this paper, we report the successful preparation of LbL films from wine alternated with BSA and an investigation of the growth regimes of the $\mathrm{BSA} /$ wine LbL film. To elucidate the exponential growth, we have used scale laws and fractal concepts to analyse the AFM results of the films.

\section{Materials and Methods}

Portuguese red wine made from Vitis vinifera L. cv. Touriga Nacional (2007 vintage) was obtained from commercial wineries. Wine was used as received without any dissolution immediately after the bottle was opened. BSA (fraction V, purity 96-100\%) from Acros Organics was used as received. The experimental film fabrication procedures are essentially the same as those described by Decher [33]. Since our experience shows that the rinsing step after the deposition of BSA layer leads to the formation of surface with a low coverage ratio, the washing step was excluded. To build the multilayers of BSA alternated with wine (hereinafter BSA/wine films), wine $(\mathrm{pH}$ 3.8) was alternated with $0.5 \mathrm{~g} / \mathrm{L}$ BSA diluted in ultrapure water. The $\mathrm{pH}$ of the BSA solution was adjusted to 7.5 (close to the physiological $\mathrm{pH}$ ) by adding appropriate amounts of ammonium hydroxide. The films were adsorbed on quartz $(36 \mathrm{~mm} \times 14 \mathrm{~mm} \times 1 \mathrm{~mm})$. The absorbance of the wine, which is proportionate to the adsorbed amount of the wine solute, was monitored by measuring the UVVis absorption spectra with a double-beam Thermo Scientific spectrophotometer, model Genesys 10 . The multilayer films were assembled by alternating the immersion of the substrate into the BSA and into the wine solution. After each deposition step, the substrates were dried under an air flow. The morphology of the films was studied with a NanoSurf Instruments atomic force microscope EasyScan II in the tapping mode $(256 \times 256$ pixels $)$ under ambient conditions. The tapping mode was selected instead of the contact mode because it is less damaging to the surfaces under investigation [34], as the transferred energy is less and the tip does not exert a significant lateral force on the surface. A sample area of $5 \mu \mathrm{m} \times 5 \mu \mathrm{m}$ was scanned, and an image was acquired. The films roughness was determined using NanoSurf Instruments software.

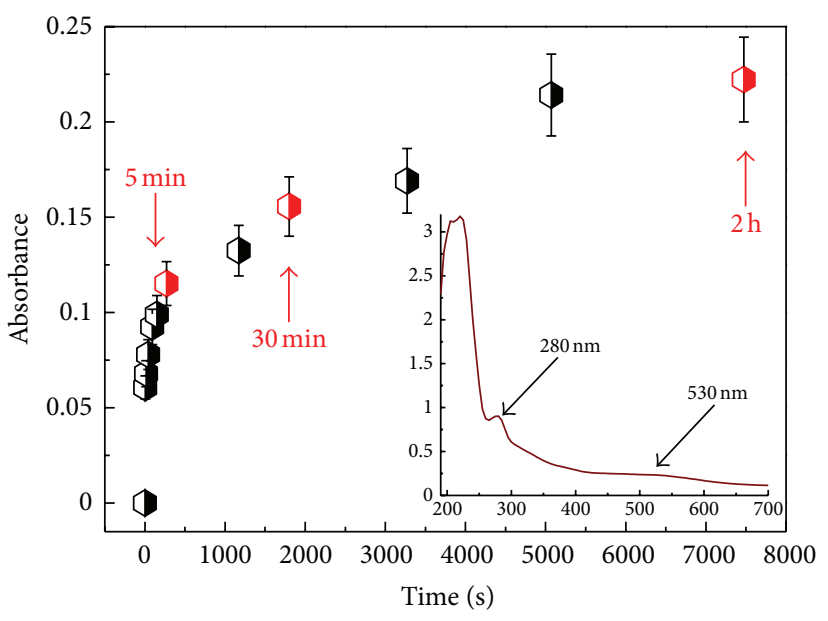

FIGURE 1: Absorbance at $530 \mathrm{~nm}$ versus time of wine adsorption for the BSA/wine layers. The inset in the figure shows the UV-vis spectra of the BSA/wine LbL film.

\section{Results and Discussion}

The inset in Figure 1 shows the spectra of a BSA/wine LbL film with bands at 280 and $530 \mathrm{~nm}$. In wines, anthocyanins absorb between 520 and $530 \mathrm{~nm}[35,36]$, and the BSA has a band at $280 \mathrm{~nm}$ due to the amino acids tyrosine and tryptophan [37]. The adsorption process was monitored via UV-Vis spectroscopy after the deposition of each bilayer onto the quartz substrate using an absorption wavelength of $530 \mathrm{~nm}$. The curve in Figure 1 was obtained after the deposition of BSA using an immersion time of 10 minutes for all bilayers. The immersion time of the films in the wine solution was varied from $5 \mathrm{~s}$ to $120 \mathrm{~min}$, and an increase in the absorbance at $530 \mathrm{~nm}$ was observed (Figure 1).

Figure 1 shows the initial formation of a plateau at about $1.5 \mathrm{~h}$. The formation of the plateau results from saturation sites, indicating formation of a complete layer. Thus, we can infer that the BSA layer will be completely filled after $2 \mathrm{~h}$ of wine immersion. In this case, we can expect the growth process of the BSA/wine film $(10 \mathrm{~min} / 2 \mathrm{~h})$ to be linear, with approximately the same amount of material being deposited in each bilayer. Further, we can expect that any other deposition time (shorter times) will lead to a less effective growth with incomplete layers.

3.1. Growth Regime of the BSA/Wine Films. An important characteristic to be studied in the growth of LbL films is the adsorbed amount at each deposition step. Figure 2 depicts the absorbance as a function of the bilayer number for the BSA/wine LbL films at different times during the wine deposition.

Based on the results shown in Figure 1, the multilayers films were assembled by alternating the immersion of the substrate into the BSA for 10 minutes and into the wine solution for $5 \mathrm{~min}, 30 \mathrm{~min}$, and $2 \mathrm{~h}$. After each deposition, the substrates were dried under an air flow. As shown in the inset (Figure 2), the adsorbed amount of mass, which is proportional to absorbance [21, 29], increases linearly with 


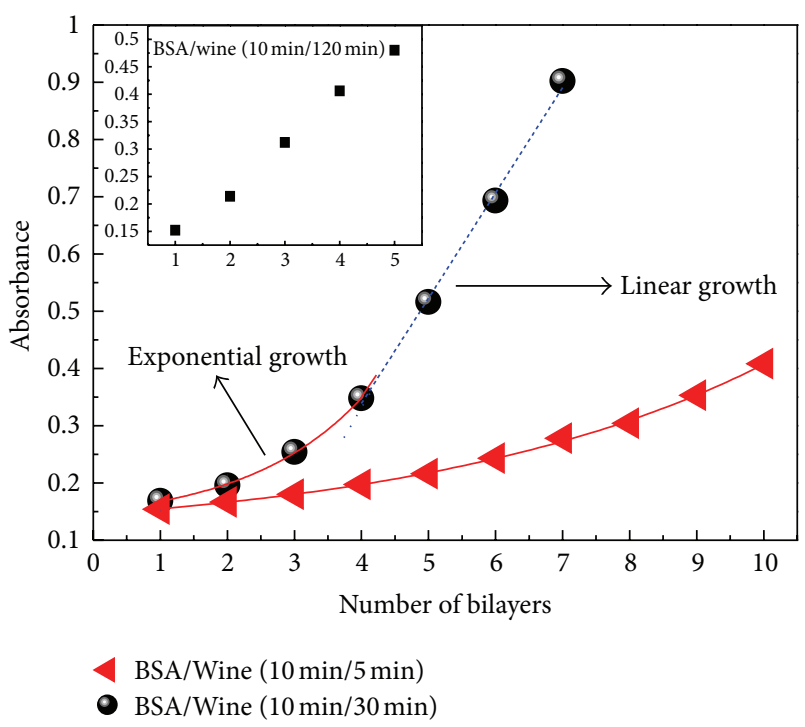

FIGURE 2: Absorbance versus number of bilayers for BSA/wine films. The solid lines are adjustments of exponential and linear equations to results $(R$-square $=0.99$ for all adjustments). The inset shows the results for BSA/wine $(10 \mathrm{~min} / 2 \mathrm{~h})$.

an increase in the number of bilayers when the deposition time in the wine is $2 \mathrm{~h}$, as expected. On the other hand, when the immersion time in the wine solution is $5 \mathrm{~min}$, the growth is exponential, and surprisingly, we found that the transition from exponential to linear growth occurs with an immersion time of 30 minutes in the wine solution. Unlike the linear growth, where the results suggest that the same amount of material was adsorbed in each deposition step, the exponential growth indicates that in each deposition cycle the adsorbed amount of material increases. This behaviour was identified in several systems and was associated with in- and out-diffusion processes [23-25, 38-41]. Recently, some authors have proposed alternative explanations for the exponential growth, which are based on surface structure models, such as island and dendritic models [42]. However, the mechanisms that give rise to the exponential growth were not well elucidated.

\subsection{Film Growth Analysis Using Dynamic Scale Laws and} Fractal Concepts. To explain the exponential and linear growth behaviour of the BSA/wine $(10 / 30 \mathrm{~min})$ films, we performed an analysis of the AFM images of these film surfaces using fractal concepts and scale laws [43]. Figure 3 shows the AFM images of the BSA/wine films for 1,3 , and 7 bilayers in which the top layer is wine.

The images were taken in a scan window of $5 \mu \mathrm{m} \times 5 \mu \mathrm{m}$. To characterise the interface quantitatively, we determined the roughness exponents, $\alpha$, using scale laws [43]. Figure 4 shows the $\log W$ versus $\log L$ plot, where $W$ is the root-meansquare (RMS) roughness and $L$ is the scan window size. In the linear region, where the scaling law $W_{\text {sat }} \propto L^{\alpha}$ is satisfied, the $\alpha$ value can be obtained from the slope of the curve, and the fractal dimension of a surface can be calculated using $D_{f}=3-\alpha[43]$.

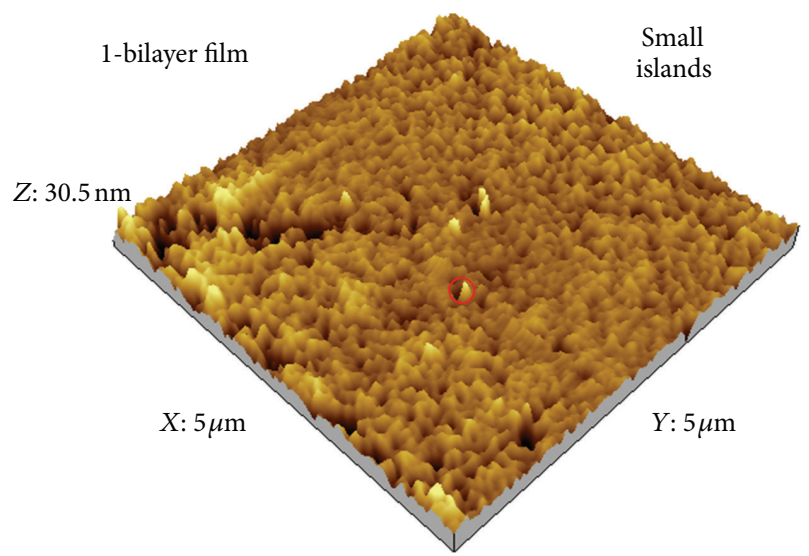

(a)

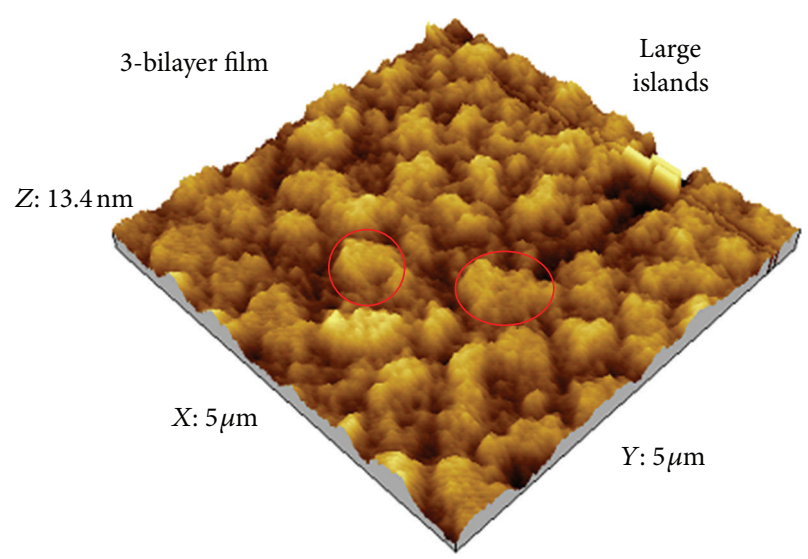

(b)

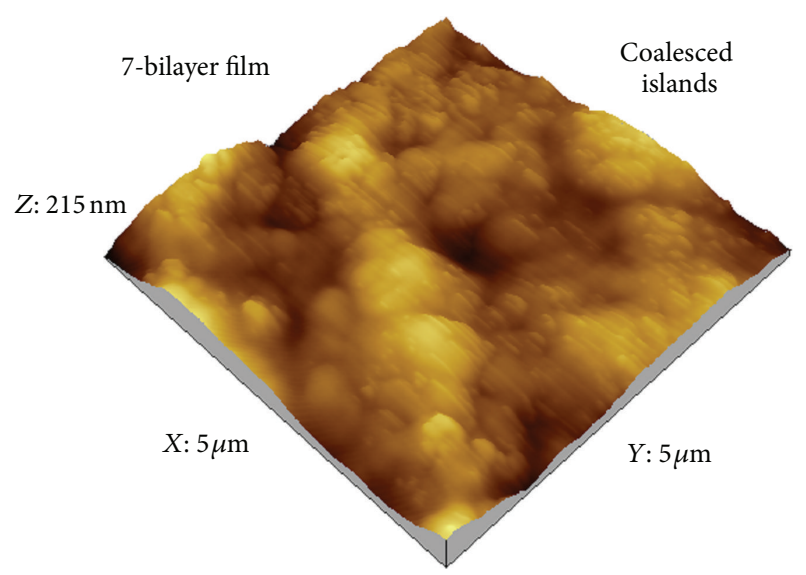

(c)

FIgURE 3: AFM images of BSA/wine LbL films with 1, 3, and 7 bilayers. Wine layer is at top. The figure shows the interface growth with small islands for 1-bilayer film, large island for 3-bilayer, and coalesced islands revealing a smooth interface (7-bilayer films).

Table 1 shows the roughness RMS along with the roughness exponent and the fractal dimension for the LbL films with 1,3 , and 7 bilayers. The roughness of the interface is defined by the RMS fluctuation in the height between the structures forming on the surface. The fractality relates to the effect of changes in the scale of observation on the roughness. 


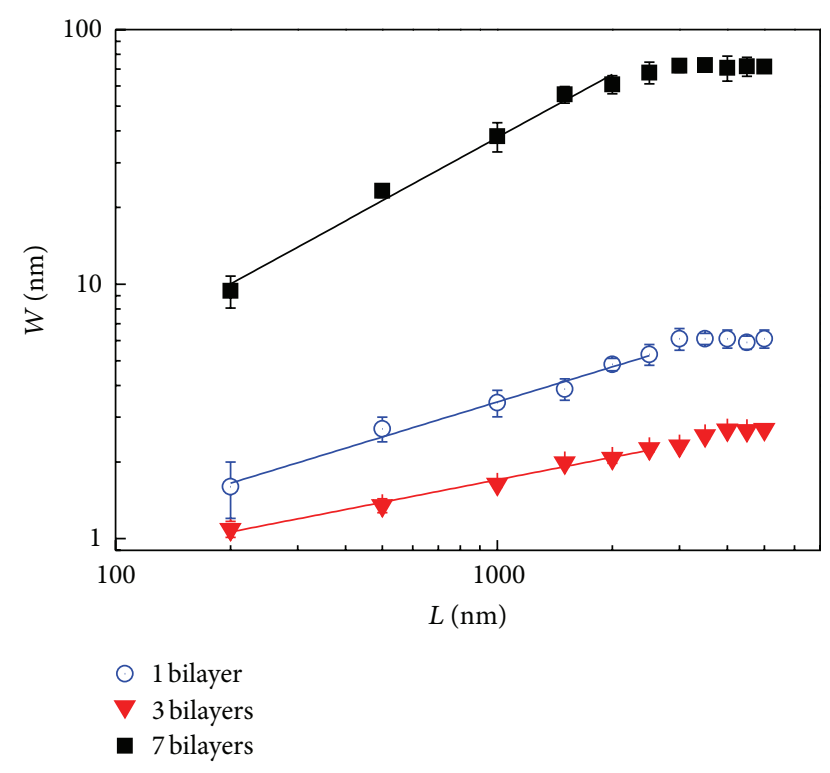

FIgURE 4: Log-log plot of RMS roughness $(W)$ versus scan window size $(L)$ for BSA/wine LbL films with 1, 3, and 7 bilayers.

TABLE 1: Root mean square (RMS) roughness, exponents, and fractal dimensions for LbB films with 1, 3, and 7 bilayers.

\begin{tabular}{lccc}
\hline Bilayer & $\begin{array}{c}\text { RMS }(\mathrm{nm}) \\
(5 \mu \mathrm{m} \times 5 \mu \mathrm{m})\end{array}$ & $\alpha$ & $\mathrm{DF}$ \\
\hline 1 & 6.1 & $0.45 \pm 0.02$ & 2.55 \\
3 & 2.7 & $0.31 \pm 0.02$ & 2.69 \\
7 & 71.6 & $0.82 \pm 0.04$ & 2.18 \\
\hline
\end{tabular}

As observed in Figure 3, small aggregates [21, 43-46] can be noted for the 1-bilayer films with whole substrate coverage. For the 3-bilayer films, there are large and small aggregates emerging from the surfaces of the films. For the 7-bilayer films, the surface is smoother compared with the 1 and 3 bilayer films. From the viewpoint of the fractal dimension, for the 1-bilayer film, the small aggregates shown in Figure 3 are associated with the fractal dimension of $D_{f} \sim 2.5$. For the 3-bilayer film, the small aggregates (revealed on the top of the larger aggregates) contribute to an increase in the fractal dimension $\left(D_{f} \sim 2.7\right)$ as compared to the 1-bilayer film. Finally, the smooth surface noted in the 7-bilayer films is related to a lower fractal dimension $\left(D_{f} \sim 2.2\right)$ among the films as a result of the coalescence of large islands, which can arise from a process of aggregation.

As shown in Figure 2, the BSA/wine $(10 / 30 \mathrm{~min})$ film has two growth regimes, exponential and linear, that can be explained in the following way: the exponential regime is associated with sub-bilayer growth, which results from the development and coalescence of islands (Figure 5(b)). These islands are aggregates that can arise from the interplay between the deposition, diffusion, aggregation, and desorption processes evolving with time until a full bilayer is formed. The linear regime is associated with bilayer deposition (with the same amount of mass deposited in each layer) of
$\mathrm{BSA} /$ wine, as it is the case with an immersion time in wine of $2 \mathrm{~h}$. The formation of island on the films is likely due to an inhomogeneous distribution of the charge density on the surface of the BSA monolayer. Sites with a higher charge density should attract more molecules and smaller aggregates than sites with a lower charge density. Our hypothesis of the sub-bilayer and bilayer growth regimes is supported by the findings of Picart et al. [23] and Lavalle et al. [25], who showed that films with islands display exponential growth.

According to the model of the nucleation and growth of islands, the deposited grains begin to coalesce, thus achieving the growth process of the film [42]. At this stage, the grains begin to fill the voids created by the deposition as described by the Kardar-Parisi-Zhang (KPZ) model, which predicts lateral growth [43] and is a model well established for studying interface growth. KPZ is one of the models used to describe stochastic formation at an interface, which can be related to the growth of a film surface [47-49]. In this model, it is assumed that the interface grows as a result of the deposition of particles and the surface relaxation produced by lateral growth and desorption. With the voids being filled, the surfaces of the grains become larger and less fractal, and this process contributes to reducing the fractality of the system. The $\alpha$ exponent in the KPZ model is close to $0.4[34,50,51]$, and this value is in good agreement with the values found for the 1- and 3-bilayer films.

For our films, the fractal dimension is ca. 2.2 for the 7bilayer films. With an increasing number of bilayers, there is a higher probability that the molecules will adsorb onto the empty sites formed at the top of the islands structures [52], as shown in the morphology for the LbL films with 1 and 3 bilayers (Figure 3), and thus, the structures are smooth. The fractal dimension is close to 2 , as $\alpha \sim 0.8$, and indicates an Euclidean surface [43]. In fact, for the 7-bilayer film, $D_{f} \sim 2$ is comparable to the values derived from the growth models incorporating surface diffusion [53], which is predicted by the stochastic differential equation of the variation in the height (h) of the interface as a function of time [43,53]:

$$
\frac{\partial h}{\partial t}=-K \nabla^{4} h+\eta(x, t) .
$$

The first term on the right-hand side of the equation represents the surface relaxation, and $n(x, t)$ represents the random fluctuations that occur during the deposition. The origin of $K$ is the surface diffusion. This equation provides a description of the morphology and dynamics of the interface at a large length scale and for long times (after several bilayers).

In summary, we propose to correlate the exponential growth with the fractality of the surfaces. In Figure 2 (BSA/wine-10/30 min), the curve indicates that for the first bilayers, the quantity of the material increases slowly. This stage may be associated with the nucleation process, which corresponds to the deposition of small grains or islands on the substrate. These small islands appear with dimensions of approximately $100 \mathrm{~nm}$, as observed in the height profile (Figure 5(a)), and generate a surface with a high fractality $\left(D_{f}=2.55\right)$. Surfaces with a high fractality may be related to the increase in area available for deposition. The surface analysed for the LbL film with 3 bilayers shows that 


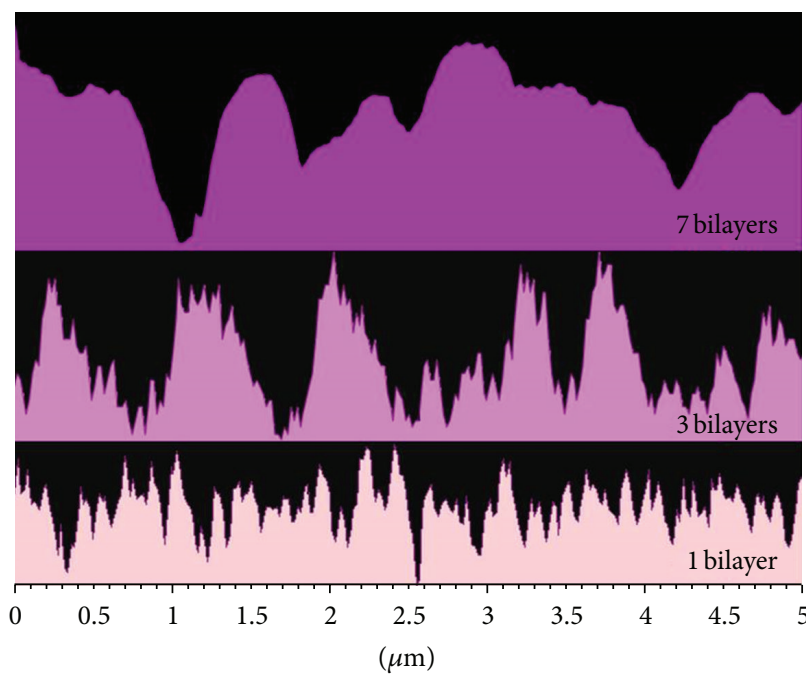

(a)

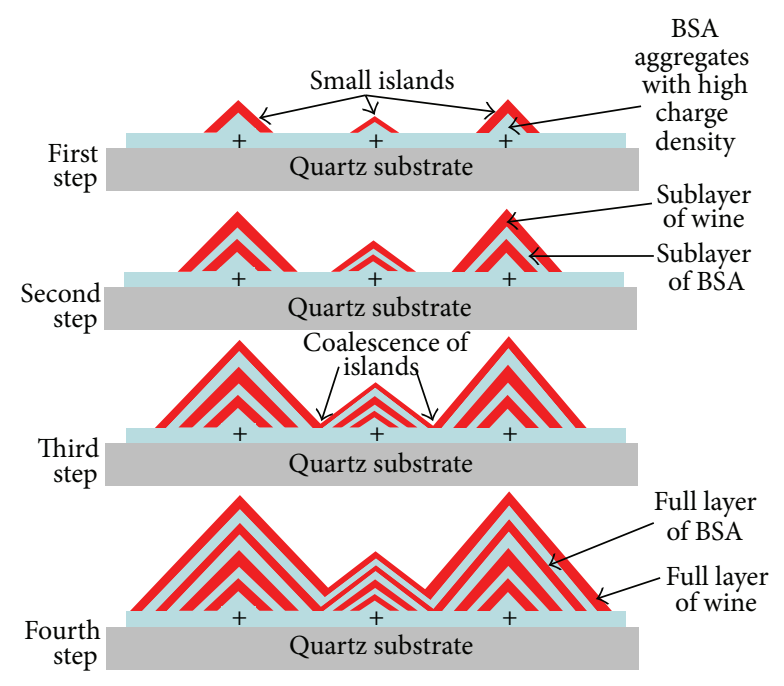

(b)

FIGURE 5: (a) Cross-section of BSA/wine films with wine atop, obtained by AFM images to show the different morphology characteristics; (b) schematic diagram showing the growth of the BSA/wine films though evolution of islands and their coalescences. Each step corresponds to immersion in BSA solution and after in wine. The smoothing of the edges of the islands by diffusion is not displayed.

the fractality further increases from 2.55 to 2.69 , indicating that the area available for deposition continues to increase, which may explain the exponential growth (Figure 2). When the islands coalesce, the deposition rate decreases, and the exponential growth transitions to linear growth [42].

\section{Conclusion}

Layer-by-layer films of wine alternated with bovine serum albumin (BSA) were obtained for the first time, demonstrating the effectiveness of the $\mathrm{LbL}$ technique. The regime of film growth depends on the time of the deposition in the wine solution. This time can be easily determined through experiments on the kinetics of the bilayers. Times shorter than necessary to completely fill the layer lead to the onset of an exponential growth regime that was characterised by the deposition of islands that are responsible for the formation of incomplete bilayer (sub-bilayers). These islands contribute to the increased fractality of the surface, which is directly related to an increase in the deposition area available. With the growth and coalescence of islands, a full bilayer is formed (linear growth).

By using scale laws and the fractal dimension, we have characterised the films and found $D_{f}=2.18$ for 7-bilayer films, which is close to the value predicted by the stochastic differential equation $\partial h / \partial t=-K \nabla^{4} h+\eta(x, t)\left(D_{f}=2\right)$. The growth for the 1- and 3-bilayer films was explained by the KPZ model, which helps in extracting general features of the system under study. Our results indicate that the use of an interface growth analysis employing scale laws and fractal concepts as well as stochastic growth equations is an effective approach for investigating LbL film growth.

Finally, the analysis in this paper contributes to the understanding of the mechanisms involved in the construction of exponential LbL multilayers and the knowledge that wine is associated with health-related benefit, LbL films from wine alternating with BSA can be used as a starting point for future investigations into processes based on the molecular interaction between wine and biological molecules that study the wine components immobile in films rather than wine in liquid phase.

\section{Acknowledgment}

This work was supported by CNPq and Capes (Brazil).

\section{References}

[1] Z. Liang, C. L. Owens, G. Y. Zhong, and L. Cheng, "Polyphenolic profiles detected in the ripe berries of Vitis vinifera germplasm," Food Chemistry, vol. 129, no. 3, pp. 940-950, 2011.

[2] Z. Liang, Y. Yang, L. Cheng, and G. -Y Zhong, "Polyphenolic composition and content in the ripe berries of wild Vitis species," Food Chemistry, vol. 132, pp. 730-738, 2012.

[3] A. Zoechling, F. Liebner, and A. Jungbauer, "Red wine: a source of potent ligands for peroxisome proliferator-activated receptor $\gamma$," Food \& Function, vol. 2, pp. 28-38, 2011.

[4] J. M. Guilford and J. M. Pezzuto, "Wine and health: a review," American Journal of Enology and Viticulture, vol. 62, pp. 471486, 2011.

[5] P. Gresele, C. Cerletti, G. Guglielmini, P. Pignatelli, G. de Gaetano, and F. Violi, "Effects of resveratrol and other wine polyphenols on vascular function: an update," Journal of Nutritional Biochemistry, vol. 22, no. 3, pp. 201-211, 2011.

[6] M. Das and D. K. Das, "Resveratrol and cardiovascular health," Molecular Aspects of Medicine, vol. 31, no. 6, pp. 503-512, 2010.

[7] P. Wang, K. Zhang, Q. Zhang, J. Mei, C. J. F. Z. Chen, and D. H. $\mathrm{Yu}$, "Effects of quercetin on the apoptosis of the human gastric carcinoma cells," Toxicology in Vitro, vol. 26, pp. 221-228, 2012. 
[8] A. R. Patel, C. P. Heussen, J. Hazekamp, E. Drost, and K. Velikov, "Quercetin loaded biopolymeric colloidal particles prepared by simultaneous precipitation of quercetin with hydrophobic protein in aqueous medium," Food Chemistry, vol. 133, pp. 423429, 2012.

[9] M. Thiruchenduran, N. A. Vijayan, J. K. Sawaminathan, and S. N. Devaraj, "Protective effect of grape seed proanthocyanidins against cholesterol cholic acid diet-induced hypercholesterolemia in rats," Cardiovascular Pathology, vol. 20, pp. 361368, 2011.

[10] H. Xiang, Y. Xie, and J. Hang, "Study on the interaction of DNA with resveratrol by resonance light scattering technique and its analytical application," Journal of Analytical Chemistry, vol. 66, pp. 618-622, 2011.

[11] G. Wang, L. Wang, W. Tang, X. Hao, Y. Wang, and Y. Lu, "Binding of quercetin to lysozyme as probed by spectroscopic analysis and molecular simulation," Journal of Fluorescence, vol. 21, pp. 1879-1886, 2011.

[12] L. Yang, P. Li, Y. Gao, and D. Wu, "Qualitative observation of chemical change rate for quercetin in basic medium characterized by time resolved UV-vis spectroscopy," Journal of Molecular Liquids, vol. 151, no. 2-3, pp. 134-137, 2010.

[13] G. Gonzalez-Neves, G. Gil, G. Favre, and M. Ferrer, "Influence of grape composition and winemaking on the anthocyanin composition of red wines of Tannat," International Journal of Food Science and Technology, vol. 47, pp. 900-909, 2012.

[14] V. Ivanova, B. Vojnoski, and M. Stefova, "Effect of winemaking treatment and wine aging on phenolic content in Vranec wines," Journal of Food Science and Technology, vol. 49, pp. 161-172, 2012.

[15] S. Fragoso, L. Aceña, J. Guasch, M. Mestres, and O. Busto, "Quantification of phenolic compounds during red winemaking using FT-MIR spectroscopy and PLS-regression," Journal of Agricultural and Food Chemistry, vol. 59, pp. 10795-10802, 2011.

[16] G. Gambacorta, D. Antonacci, S. Pati et al., "Influence of winemaking technologies on phenolic composition of Italian red wines," European Food Research and Technology, vol. 233, pp. 1057-1066, 2011.

[17] A. Baiano, C. Terracone, G. Gambacorta, and E. La Notte, "Phenolic content and antioxidant activity of primitivo wine: comparison among winemaking technologies," Journal of Food Science, vol. 74, no. 3, pp. C258-C267, 2009.

[18] O. Cala, N. Pinaud, C. Simon et al., "NMR and molecular modeling of wine tannins binding to saliva proteins: revisiting astringency from molecular and colloidal prospects," The FASEB Journal, vol. 24, no. 11, pp. 4281-4290, 2010.

[19] R. Pradelles, D. Chassagne, S. Vichi, R. Gougeon, and H. Alexandre, "(-)Geosmin sorption by enological yeasts in model wine and FTIR spectroscopy characterization of the sorbent," Food Chemistry, vol. 120, no. 2, pp. 531-538, 2010.

[20] G. Decher and J. B. Schlenoff, Multilayer Thin Films: Sequential Assembly of Nanocomposite Materials, Wiley-VCH, 2003.

[21] J. B. Brito, D. J. C. Gomes, V. D. Justina et al., "Nanostructured films from phthalocyanine and carbon nanotubes: surface morphology and electrical characterization," Journal of Colloid and Interface Science, vol. 367, pp. 467-471, 2012.

[22] D. L. Elbert, C. B. Herbert, and J. A. Hubbell, “Thin polymer layers formed by polyelectrolyte multilayer techniques on biological surfaces," Langmuir, vol. 15, no. 16, pp. 5355-5362, 1999.

[23] C. Picart, P. Lavalle, P. Hubert et al., "Buildup mechanism for poly(L-lysine)/hyaluronic acid films onto a solid surface," Langmuir, vol. 17, no. 23, pp. 7414-7424, 2001.
[24] C. Picart, J. Mutterer, L. Richert et al., "Molecular basis for the explanation of the exponential growth of polyelectrolyte multilayers," Proceedings of the National Academy of Sciences of the United States of America, vol. 99, no. 20, pp. 12531-12535, 2002.

[25] P. Lavalle, C. Gergely, F. J. G. Cuisinier et al., "Comparison of the structure of polyelectrolyte multilayer films exhibiting a linear and an exponential growth regime: an in situ atomic force microscopy study," Macromolecules, vol. 35, no. 11, pp. 44584465, 2002.

[26] L. Richert, P. Lavalle, E. Payan et al., "Layer by layer buildup of polysaccharide films: physical chemistry and cellular adhesion aspects," Langmuir, vol. 20, no. 2, pp. 448-458, 2004.

[27] P. Kujawa, P. Moraille, J. Sanchez, A. Badia, and F. M. Winnik, "Effect of molecular weight on the exponential growth and morphology of hyaluronan/chitosan multilayers: a surface plasmon resonance spectroscopy and atomic force microscopy investigation," Journal of the American Chemical Society, vol. 127, no. 25, pp. 9224-9234, 2005.

[28] F. Boulmedais, V. Ball, P. Schwinte, B. Frisch, P. Schaaf, and J. C. Voegel, "Buildup of exponentially growing multilayer polypeptide films with internal secondary structure," Langmuir, vol. 19, no. 2, pp. 440-445, 2003.

[29] P. Lavalle, J. C. Voegel, D. Vautier, B. Senger, P. Schaaf, and V. Ball, "Dynamic aspects of films prepared by a sequential deposition of species: perspectives for smart and responsive materials," Advanced Materials, vol. 23, no. 10, pp. 1191-1221, 2011.

[30] P. Ribéreau-Gayon, Handbook of Enology : The Chemistry of Wine Stabilization and Treatments, vol. 2, John Wiley \& Sons, 2nd edition, 2000.

[31] A. E. Hagerman, M. E. Rice, and N. T. Ritchard, "Mechanisms of protein precipitation for two tannins, pentagalloyl glucose and epicatechin16 $(4 \rightarrow 8)$ catechin (procyanidin)," Journal of Agricultural and Food Chemistry, vol. 46, no. 7, pp. 2590-2595, 1998.

[32] C. Simon, K. Barathieu, M. Laguerre et al., "Three-dimensional structure and dynamics of wine tannin-saliva protein complexes. A multitechnique approach," Biochemistry, vol. 42, no. 35, pp. 10385-10395, 2003.

[33] G. Decher, "Fuzzy nanoassemblies: toward layered polymeric multicomposites," Science, vol. 277, no. 5330, pp. 1232-1237, 1997.

[34] N. C. De Souza, M. Ferreira, K. Wohnrath, J. R. Silva, O. N. Oliveira Jr., and J. A. Giacometti, "Morphological characterization of Langmuir-Blodgett films from polyaniline and a ruthenium complex (Rupy): influence of the relative concentration of Rupy," Nanotechnology, vol. 18, no. 7, Article ID 075713, 2007.

[35] I. G. Roussis, I. Lambropulos, and K. Soulti, "Scavenging capacities of some wines and wine phenolic extracts," Food Technology and Biotechnology, vol. 43, no. 3, pp. 351-358, 2005.

[36] C. Papadopoulou, K. Soulti, and I. G. Roussis, "Potential antimicrobial activity of red and white wine phenolic extracts against strains of staphylococcus aureus, escherichia coli and Candida albicans," Food Technology and Biotechnology, vol. 43, no. 1, pp. 41-46, 2005.

[37] A. L. Lehninger, Princípios De Bioquímica, Sarvier, 3rd edition, 2002.

[38] C. Porcel, P. Lavalle, V. Ball et al., "From exponential to linear growth in polyelectrolyte multilayers," Langmuir, vol. 22, no. 9, pp. 4376-4383, 2006.

[39] E. Guzmán, R. Chuliá-Jordán, F. Ortega, and R. G. Rubio, "Influence of the percentage of acetylation on the assembly of 
LbL multilayers of poly(acrylic acid) and chitosan," Physical Chemistry Chemical Physics, vol. 13, pp. 18200-18207, 2011.

[40] C. Peng, Y. S. Thio, R. A. Gerhardt, H. Ambaye, and V. Lauter, "pH-promoted exponential layer-by-layer assembly of bicomponent polyelectrolyte/nanoparticle multilayers," Chemistry of Materials, vol. 23, pp. 4548-4556, 2011.

[41] P. Bieker and M. Schönhoff, "Linear and exponential growth regimes of multilayers of weak polyelectrolytes in dependence on pH," Macromolecules, vol. 43, pp. 5052-5059, 2010.

[42] D. T. Haynie, E. Cho, and P. Waduge, "In and out diffusion' hypothesis of exponential multilayer film buildup revisited," Langmuir, vol. 27, no. 9, pp. 5700-5704, 2011.

[43] A. L. Barabási and H. E. Stanley, Fractal Concepts in Surface Growth, Cambridge University Press, Cambridge, UK, 1995.

[44] R. C. Salvarezza, L. Vázquez, P. Herrasti, P. Ocón, J. M. Vara, and A. J. Arvia, "Self-affine fractal vapour-deposited gold surfaces characterization by scanning tunnelling microscopy," Europhysics Letters, vol. 20, no. 8, article 727, 1992.

[45] P. Keblinski, A. Maritan, F. Toigo, R. Messier, and J. R. Banavar, "Continuum model for the growth of interfaces," Physical Review E, vol. 53, no. 1, pp. 759-778, 1996.

[46] A. Mazor, D. J. Srolovitz, P. S. Hagan, and B. G. Bukiet, "Columnar growth in thin films," Physical Review Letters, vol. 60, no. 5, pp. 424-427, 1988.

[47] D. M. Aurongzeb, "Nonlinearities in sedimentation: a microscopic study," Solid State Communications, vol. 134, no. 3, pp. 165-169, 2005.

[48] C. Oguey and N. Rivier, "Roughness and scaling in cellular patterns: analysis of a simple model," Journal of Physics A, vol. 34, no. 32, article 6225, 2001.

[49] P. Neogi, "Length scales and roughness on a growing solid surface: a review," Journal of Electroanalytical Chemistry, vol. 595, pp. 1-10, 2006.

[50] N. Happo, M. Fujiwara, M. Iwamatsu, and K. Horii, "Atomic force microscopy study of self-affine fractal roughness of porous silicon surfaces," Japanese Journal of Applied Physics, vol. 37, no. 7, pp. 3951-3953, 1998.

[51] A. E. Lita and J. E. Sanchez Jr., "Effects of grain growth on dynamic surface scaling during the deposition of Al polycrystalline thin films," Physical Review B, vol. 61, pp. 7692-7699, 2000.

[52] N. C. De Souza, V. Zucolotto, J. R. Silva et al., "Morphology characterization of layer-by-layer films from PAH/MA-coDR13: the role of film thickness," Journal of Colloid and Interface Science, vol. 285, no. 2, pp. 544-550, 2005.

[53] L. Vázquez, J. M. Albella, R. C. Salvarezza, A. J. Arvia, R. A. Levy, and D. Perese, "Roughening kinetics of chemical vapor deposited copper films on Si(100)," Applied Physics Letters, vol. 68, no. 9, article 1285, 1996. 

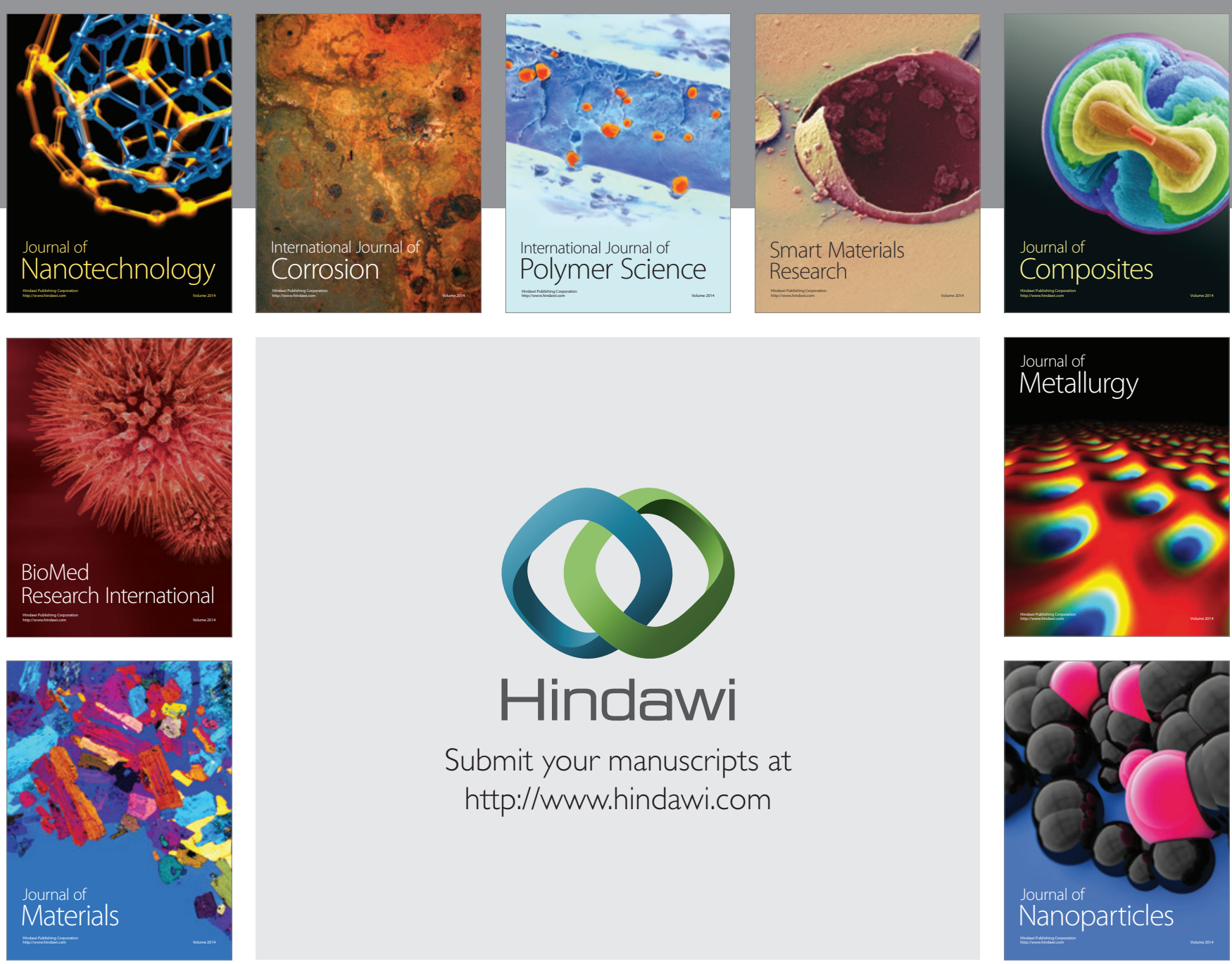

Submit your manuscripts at http://www.hindawi.com
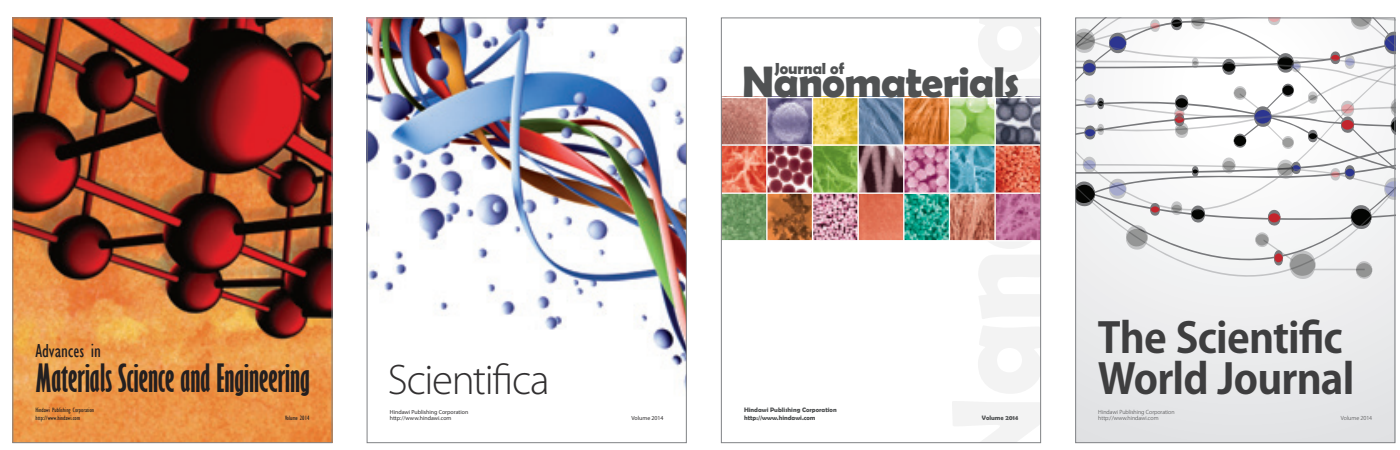

\section{The Scientific World Journal}
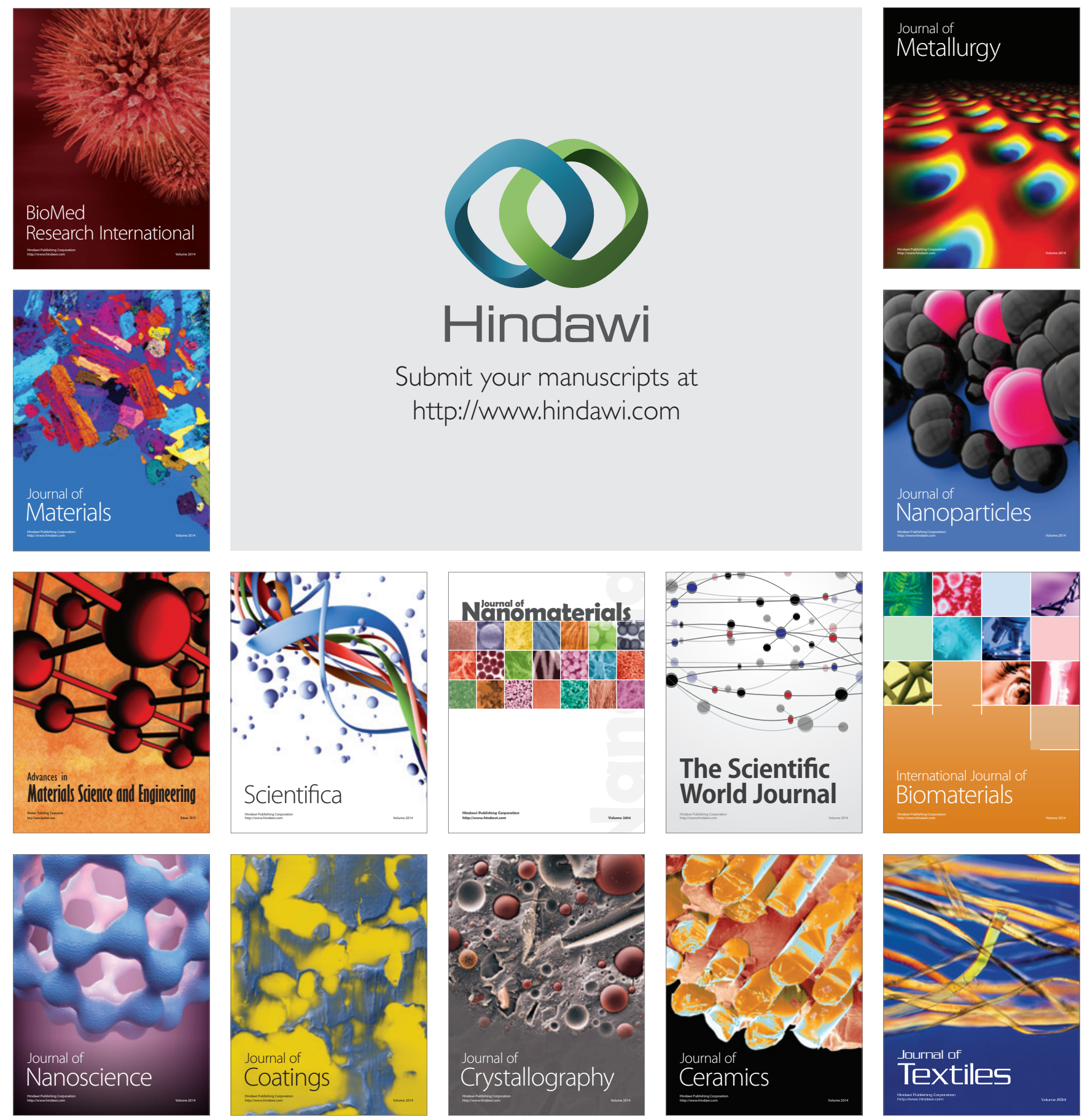